\title{
Contribution à l'étude de la binarité des étoiles de type Am
}

\author{
I. HD 125273, binaire spectroscopique à raies doubles ${ }^{\star}$ \\ HD 125273, a double-lined spectroscopic binary
}

\author{
N. Ginestet et J.-M. Carquillat
}

Laboratoire d'Astrophysique (UMR 5572), Observatoire Midi-Pyrénées, 14 avenue Edouard Belin, 31400 Toulouse, France e-mail: Carquillat@obs-mip.fr, Ginestet@obs-mip.fr

Reçu le 25 mars; accepté le 17 décembre 1997

\begin{abstract}
We present an observing program undertaken at Observatoire de Haute-Provence, for the search and study of spectroscopic binaries among Am stars. HD 125273 was recognized as an Am star some time ago (Olsen 1980; Abt 1984); observations, carried out with the CORAVEL spectrovelocimeter, show that this star is a double-lined spectroscopic binary; its orbital elements are as follows: $P=7.482664$ days; $T=2448665.660$ $\mathrm{JD} ; \omega_{1}=355.3^{\circ} ; e=0.071 ; K_{1}=49.82 \mathrm{~km} / \mathrm{s} ; K_{2}=$ $51.75 \mathrm{~km} / \mathrm{s} ; V_{0}=-17.42 \mathrm{~km} / \mathrm{s} ; a_{1} \sin i=5.113 \mathrm{Gm}$; $a_{2} \sin i=5.311 \mathrm{Gm} ; M_{1} \sin ^{3} i=0.412 M_{\odot} ; M_{2} \sin ^{3} i=$ $0.396 M_{\odot}$.

Strömgren photometry of the star (Olsen 1983) permits us to estimate the following mean physical parameters for the components of the binary: $T_{\text {eff }}=7500 \mathrm{~K}$; log $g=3.70 ; M_{v}=1.24 ; M=2.1 M_{\odot} ; R=3.2 R_{\odot}$, and to specify the evolutionary status of the system.

Assuming the above mass for the components, we found $i \sim 35^{\circ}$ and $a \sim 26 R_{\odot}$. This system is thus a detached one without possibility of eclipses. It is likely that the two components are Am stars of very close characteristics.

The $v \sin i$ values, determined from the correlation dips given by CORAVEL, are near $11 \mathrm{~km} / \mathrm{s}$ for both components. We retain for this binary the hypothesis of rotationrevolution synchronism, because it leads to a value of the radius of the stars in agreement both with the above value and the results of a previous study by Kitamura \& Kondo (1978) of similar, but eclipsing, systems. The ratio of the correlation dip areas indicates a magnitude difference $\Delta m_{v} \sim 0.2 \mathrm{mag}$. As expected in detached systems of main sequence stars, such as Am's, HD 125273 obeys the mass-luminosity law.
\end{abstract}

Key words: : stars: individual: HD 125273; binaries: spectroscopic - stars: fundamental parameters

\footnotetext{
* Étude effectuée à partir d'observations faites à
} l'Observatoire de Haute-Provence.

\section{Introduction}

La détection de systèmes binaires parmi les objets à spectre particulier (étoiles Wolf-Rayet, Am et Ap, à spectre composite, à baryum, symbiotiques, etc.) et l'étude statistique des caractéristiques de ces systèmes ont conduit à de nombreux travaux. En ce qui concerne les étoiles de type Am, et plus généralement les étoiles $\mathrm{A}$, diverses contributions ont été publiées : une investigation effectuée par Abt (1961), sur un échantillon de 25 étoiles brillantes, conclut que toutes les étoiles Am appartiennent à des systèmes binaires spectroscopiques (BS) ; d'autres études traitent de la répartition des périodes orbitales de ces objets (Abt 1965; Abt \& Bidelman 1969; Ginestet et al. 1982; Abt \& Levy 1985) : les étoiles Am appartiennent, typiquement, à des systèmes à courte période ( $<100$ jours), mais on en trouve aussi dans des systèmes à longue période (y compris des binaires visuelles). Dans leur article de 1985, Abt \& Levy reprennent l'étude initiale de 1961 avec un échantillon plus conséquent de 60 étoiles (parmi lesquelles ils trouvent près de $30 \%$ de BS à deux spectres !) et émettent plusieurs hypothèses pour expliquer la présence d'étoiles Am non seulement au sein de BS à courte période mais également, comme nous l'avons déjà mentionné, dans des systèmes binaires visuels, voire parmi les étoiles simples.

La question du synchronisme entre rotation axiale et révolution orbitale, qui revêt ici un intérêt majeur étant donné le lien qui paraît établi entre faible vitesse de rotation et phénomène Am (Michaud et al. 1983), a également fait l'objet de plusieurs publications (Abt \& Hudson 1971; Narai 1971; Abt 1975; Kitamura \& Kondo 1978). A noter que le travail de Kitamura \& Kondo traite aussi des masses et rayons des Am, de leur état d'évolution ainsi que de la relation entre métallicité et rotation ; néanmoins l'échantillon sur lequel est basé cette intéressante investigation était nécessairement restreint, car limité aux systèmes pour lesquels les données utiles étaient disponibles, 
en l'occurence $23 \mathrm{BS}$ dont la moitié sont connues comme étant des binaires à éclipses. Signalons aussi :

- un bilan de Abt \& Snowden (1973) relatif à la binarité des étoiles Ap : il se traduit, à l'inverse des Am, par une déficience en BS, du moins pour le groupe le plus important des Ap à Si et $\mathrm{Sr}-\mathrm{Cr}-\mathrm{Eu}$,

- un constat de Conti \& Barker (1973), portant sur seulement cinq étoiles de l'amas de Coma Berenice, qui semble mettre en défaut la conclusion de Abt (1961) concernant la binarité de toutes les étoiles Am.

Mais, en dépit de ce grand intérêt manifesté pour les étoiles Am, il apparaît que beaucoup de ces objets n'ont pas encore été étudiés du point de vue des vitesses radiales, donc de la binarité : à titre d'exemple, le catalogue de Hauck (1986) répertoriait 1805 étoiles Am et dans le catalogue de binaires spectroscopiques de Batten et al. (1989), sur 1469 orbites, seulement 78 concernaient des étoiles Am alors qu'elles sont supposées être toutes binaires ! Avec le dernier catalogue de Hauck (1992), le nombre d'étoiles reconnues Am s'est encore accru de $19 \%$ (2152 objets en tout !). Tout ceci nous a incités à entreprendre un programme d'observations systématiques à l'instrument CORAVEL (Baranne et al. 1979) des étoiles signalées Am qui ne figuraient pas dans les catalogues d'étoiles binaires spectroscopiques d'orbite connue : catalogue de Batten et al. (1989) et fichier des BS de l'Observatoire de Toulouse (CDS).

Notons cependant que seules les étoiles Am les plus métalliques, donc les plus typiques, sont accessibles à CORAVEL pour la mesure de leur vitesse radiale.

\section{Constitution de l'échantillon et premiers résultats}

Notre échantillon a été constitué, dans sa quasi totalité, à partir du Catalogue de Hauck (1986) ; nous avons sélectionné, au départ, les étoiles de l'Hémisphère Nord dont le type spectral des raies métalliques était F2, ou un type plus froid. Quelques étoiles Am de la liste de Bidelman (1988) ont aussi été incluses. Ce tri nous a conduit à retenir, dans un premier temps, 230 étoiles qui n'avaient pas d'orbite spectroscopique connue. A ce jour, sur 150 objets qui ont été observés, 72 ont du être exclus car ils ne donnaient pas de "pic" de corrélation à CORAVEL, ce que l'on peut sans doute attribuer soit à une métallicité surestimée, soit à une vitesse de rotation axiale trop élevée, ou encore à une combinaison de ces deux éléments. Parmi les 78 étoiles restantes, près de $60 \%$ sont des binaires spectroscopiques : 25 ont maintenant des périodes déterminées et 20 ont une vitesse radiale (VR) variable ; 21 autres paraissent avoir une VR constante mais doivent encore être surveillées car il n'est pas exclu que certaines d'entre elles soient des BS à longue période. Enfin 12 étoiles ont encore trop peu de mesures de VR pour que l'on puisse se prononcer sur leur binarité.

Les observations sont effectuées à l'Observatoire de Haute-Provence, avec le télescope suisse de $1 \mathrm{~m}$ sur lequel est monté CORAVEL. Cet article présente le premier résultat de cette investigation et concerne le système HD 125273.

\section{Etude de HD $125273\left(=\mathrm{BD}+53^{\circ} 1704\right)$}

La nature Am de HD 125273, étoile de magnitude 7,8 dans la constellation du Bouvier, a d'abord été suspectée par Olsen (1980) sur la base d'observations photométriques dans le système de Strömgren, puis confirmée et précisée par les observations spectrographiques de Abt (1984) qui donne la classification détaillée de cette étoile suivant la raie $\mathrm{K}$ de $\mathrm{Ca}$ II, les raies de l'hydrogène et les raies métalliques, soit : A6/F1/F3.

\subsection{Vitesses radiales}

Aucune vitesse radiale de HD 125273 n'était, à notre connaissance, publiée. Dès nos premières observations, en février 1992, nous avons constaté que les traces de corrélation des deux composantes de cette BS etaient nettement visibles ; de plus, la différence de leurs profondeurs permettait de les relier sans ambiguïté aux composantes primaire ou secondaire. Nos observations, $47 \mathrm{VR}$ pour la primaire et $41 \mathrm{VR}$ pour le compagnon, poursuivies jusqu'en janvier 1997 (240 cycles orbitaux), ont permis la détermination des éléments orbitaux définitifs de cette BS à raies doubles de 7,5 jours de période. Le traitement des observations (obtention des VR définitives rattachées au système international ; déconvolution des traces de corrélation) a été effectué à l'Observatoire de Genève. La précision des VR (erreur standard interne moyenne) est de $0,7 \mathrm{~km} / \mathrm{s}$ pour celles de la composante primaire, et de $0,8 \mathrm{~km} / \mathrm{s}$ pour celles de la secondaire.

\subsection{Calcul des éléments orbitaux}

Le calcul des éléments orbitaux de cette BS a été effectué au Laboratoire d'Astrophysique de l'Observatoire midiPyrénées, sur ordinateur Dec Alpha, avec nos programmes FORTRAN BS1 et BS2 (Nadal et al. 1979). Dans un premier temps, au moyen du programme BS1, nous avons calculé séparément ces éléments pour les composantes primaire et secondaire afin de nous assurer qu'ils étaient en concordance et qu'il n'y avait donc pas de perturbations physiques dans le système. Ensuite, les éléments définitifs ont été déterminés à l'aide du programme BS2 qui traite globalement les VR des composantes d'une BS à deux spectres. Pour effectuer ce calcul, compte tenu de leurs erreurs standards moyennes, les VR ont été pondérées avec les valeurs 1 pour la primaire et $3 / 4$ pour la secondaire ; une VR du compagnon a cependant été pondérée à la valeur $1 / 2$ car son erreur standard interne 


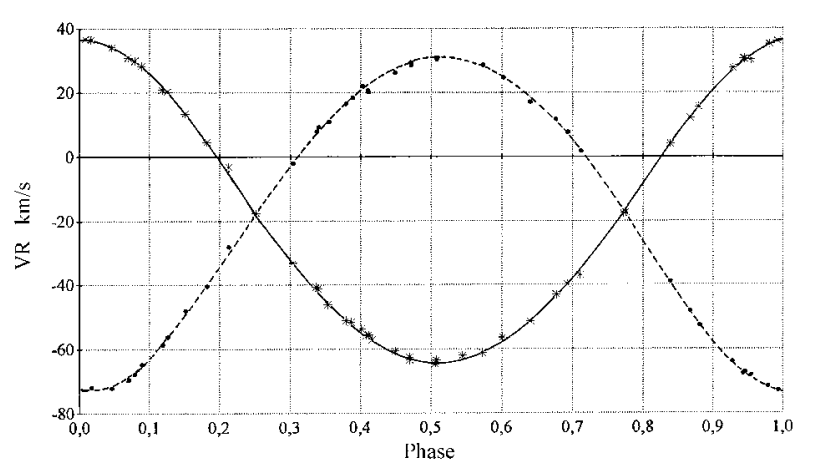

Fig. 1. Courbes de vitesse radiale des composantes primaire (trait plein) et secondaire (trait interrompu) de la binaire spectroscopique HD 125273. La phase zéro correspond à l'époque du passage au périastre

dépassait $1 \mathrm{~km} / \mathrm{s}$. Les éléments orbitaux de HD 125273 ainsi obtenus sont les suivants :

$P=7,482664 \pm 0,000047$ jours

$T=\mathrm{JJ} 2448665,660 \pm 0,042$

$\omega_{1}=355,3^{\circ} \pm 2,0^{\circ}$

$e=0,071 \pm 0,003$

$K_{1}=49,82 \pm 0,15 \mathrm{~km} / \mathrm{s}$

$K_{2}=51,75 \pm 0,18 \mathrm{~km} / \mathrm{s}$

$V_{0}=-17,42 \pm 0,09 \mathrm{~km} / \mathrm{s}$

$a_{1} \sin i=5,113 \pm 0,015 \mathrm{Gm}$

$a_{2} \sin i=5,311 \pm 0,019 \mathrm{Gm}$

$M_{1} \sin ^{3} i=0,412 \pm 0,003 M_{\odot}$

$M_{2} \sin ^{3} i=0,396 \pm 0,003 M_{\odot}$

$\sigma(\mathrm{O}-\mathrm{C})=0,7 \mathrm{~km} / \mathrm{s}$.

Les vitesses radiales observées et les écarts $\mathrm{O}-\mathrm{C}$ figurent en Table 1 ; les courbes de VR des deux composantes, calculées à partir des éléments orbitaux ci-dessus, sont données à la Fig. 1.

\subsection{Discussion}

\section{- Paramètres physiques fondamentaux du système}

Olsen (1983) a publié les indices photométriques dans le système de Strömgren de HD $125273: b-y=$ 0,$163 ; m_{1}=0,245 ; c_{1}=0,849 ; V=7,843$. Comme $\beta$ n'était pas donné, nous l'avons déterminé à l'aide de la relation $\beta / b-y$ donnée par Crawford (1979), soit : $\beta=2,783$.

Toujours à l'aide des calibrations de Crawford (1979) pour les étoiles A de la séquence d'âge zéro, on trouve : $\delta m_{1}=-0,049 ; \sigma c_{1}=0,103$ et, au moyen de la toute récente calibration proposée par North et al. (1997), $M_{v}=$ 1,24 mag. En utilisant la grille de Moon \& Dworetsky

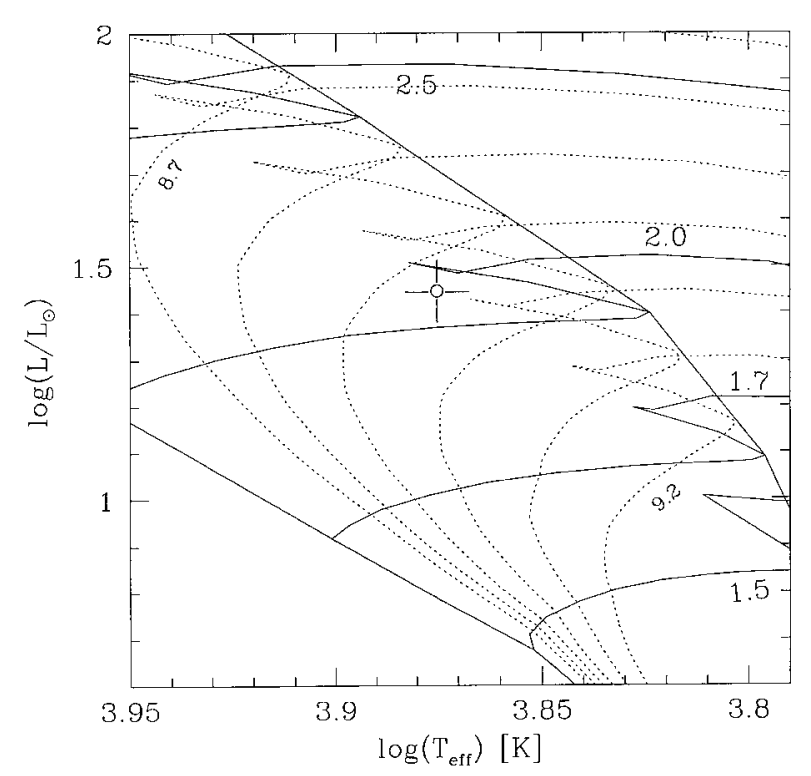

Fig. 2. Position de HD 125273 dans le diagramme HR (cercle clair). Les lignes continues sont les tracés évolutifs pour des masses comprises entre 1,5 et $2,5 M_{\odot}$; les lignes en trait interrompu représentent les isochrones, avec un pas de 0,1 en $\log t$ ( $t$ en années)

(1985) relative aux étoiles avec $T_{\text {eff }}<8500 \mathrm{~K}$ (ce qui est certainement le cas ici vu la classification de Abt et l'indice de couleur $B-V=0,29$ ), on déduit $T_{\text {eff }}=7500 \mathrm{~K}, \log$ $g=3,87$, cette dernière valeur étant ramenée à $\log g=$ 3,70 après correction de l'effet de métallicité (Dworetsky $\&$ Moon 1986) ; et par suite $M_{\mathrm{bol}}=1,16 ; \log L / L_{\odot}=$ 1,$436 ; R=3,22 R_{\odot}$.

Bien entendu ces valeurs sont des moyennes pour les deux composantes (comme les indices de Strömgren dont elles proviennent), mais cela ne présente pas un gros inconvénient étant donné que ces composantes, de masses voisines, sont certainement très semblables.

A la Fig. 2, nous montrons la position du système dans le diagramme HR, par rapport aux trajets évolutifs pour diverses masses stellaires (Schaller et al. 1992). Nous constatons que HD 125273, avec un âge de 0,85 milliard d'années $(\log t=8,93)$ apparaît quelque peu évoluée sur la séquence principale, et que la masse moyenne des composantes peut être estimée à $2,1 M_{\odot}$.

Cet état d'évolution implique $\log g=3,75$, ce qui est en excellent accord avec la valeur donnée par la photométrie. Avec $M=2,1 M_{\odot}$, on a $i \sim 35^{\circ}$, et par suite $a=a_{1}+a_{2}=18,17 \mathrm{Gm} \sim 26 R_{\odot}$.

Dans ces conditions, HD 125273 serait un système détaché ne présentant pas d'éclipses. Quant à sa distance, elle peut être estimée : 1) à partir de $M_{v}$ obtenue par la photométrie de Strömgren, et 2) à partir de la parallaxe $(\pi=3,17 \pm 0,71$ mas $)$ fournie par Hipparcos. En supposant ici que les composantes sont identiques, et par conséquent plus faibles de 0,75 mag. que la magnitude apparente $V$ du système, on trouve, avec $M_{v}=1,24$, une 
Tableau 1. Vitesses radiales de $\mathrm{HD} 125273 . \mathrm{VR}_{1},(\mathrm{O}-\mathrm{C})_{1}$ : vitesses radiales et résidus pour la composante primaire $; \mathrm{VR}_{2}$, $(\mathrm{O}-\mathrm{C})_{2}$ : mêmes quantités pour la secondaire

\begin{tabular}{|c|c|c|c|c|c|c|c|}
\hline $\mathrm{N}^{\circ}$ & Date TU & $\begin{array}{c}\text { Date JJ } \\
2400000+\end{array}$ & Phase & $\begin{array}{l}\mathrm{VR}_{1} \\
\mathrm{~km} / \mathrm{s}\end{array}$ & $\begin{array}{c}(\mathrm{O}-\mathrm{C})_{1} \\
\mathrm{~km} / \mathrm{s}\end{array}$ & $\begin{array}{l}\mathrm{VR}_{2} \\
\mathrm{~km} / \mathrm{s}\end{array}$ & $\begin{array}{c}(\mathrm{O}-\mathrm{C})_{2} \\
\mathrm{~km} / \mathrm{s}\end{array}$ \\
\hline 1 & 23,174 fev. 1992 & 48675,674 & 0,338 & $-40,8$ & $+0,7$ & $+7,9$ & $+0,3$ \\
\hline 2 & 24,170 & 48676,670 & 0,471 & $-63,4$ & $-1,1$ & $+28,6$ & $-0,6$ \\
\hline 3 & 12,258 dec. & 48968,758 & 0,507 & $-64,8$ & $-1,1$ & $+30,8$ & $+0,2$ \\
\hline 4 & 13,258 & 48969,758 & 0,640 & $-51,5$ & $+0,6$ & $+17,2$ & $-1,4$ \\
\hline 5 & 14,247 & 48970,747 & 0,772 & $-17,7$ & $+0,3$ & & \\
\hline 6 & 13,201 jan. 1993 & 49000,701 & 0,776 & $-17,4$ & $-0,4$ & & \\
\hline 7 & 1,432 juin & 49140,432 & 0,450 & $-60,7$ & $-0,2$ & $+26,3$ & $-1,1$ \\
\hline 8 & 2,359 & 49141,359 & 0,574 & $-61,3$ & $-0,1$ & $+28,7$ & $+0,7$ \\
\hline 9 & 3,075 & 49141,575 & 0,602 & $-56,5$ & $+1,5$ & $+24,7$ & $-0,1$ \\
\hline 10 & 3,389 & 49142,389 & 0,711 & $-37,1$ & $-1,0$ & $+1,7$ & $-0,2$ \\
\hline 11 & 4,345 & 49143,345 & 0,839 & $+3,9$ & $+0,7$ & $-39,0$ & $-0,2$ \\
\hline 12 & 5,057 & 49143,557 & 0,867 & $+11,9$ & $+0,1$ & $-48,1$ & $-0,3$ \\
\hline 13 & 5,394 & 49144,394 & 0,979 & & & $-71,6$ & $-0,2$ \\
\hline 14 & 5,414 & 49144,414 & 0,982 & $+35,0$ & $+0,2$ & & \\
\hline 15 & 6,115 & 49144,615 & 0,009 & $+36,5$ & $+0,6$ & & \\
\hline 16 & 6,443 & 49145,443 & 0,119 & $+20,7$ & $-1,1$ & $-58,6$ & $-0,4$ \\
\hline 17 & 7,438 & 49146,438 & 0,252 & $-17,6$ & $+0,0$ & & \\
\hline 18 & 8,390 & 49147,390 & 0,379 & $-51,2$ & $-0,8$ & $+16,5$ & $-0,3$ \\
\hline 19 & 9,118 & 49147,618 & 0,410 & $-55,8$ & $-0,2$ & $+20,6$ & $-1,7$ \\
\hline 20 & 9,356 & 49148,356 & 0,509 & $-63,5$ & $+0,2$ & $+30,8$ & $+0,2$ \\
\hline 21 & 28,235 nov. & 49319,735 & 0,412 & $-55,7$ & $+0,3$ & $+20,2$ & $-2,4$ \\
\hline 22 & 29,230 & 49320,730 & 0,545 & $-62,1$ & $+0,9$ & & \\
\hline 23 & 2,228 dec. & 49323,728 & 0,946 & $+30,4$ & $-0,0$ & $-67,1$ & $+0,0$ \\
\hline 24 & 3,229 & 49324,729 & 0,079 & $+29,8$ & $-0,3$ & $-67,7$ & $-1,0$ \\
\hline 25 & 4,231 & 49325,731 & 0,213 & $-3,2$ & $+2,2$ & $-28,1$ & $+1,8$ \\
\hline 26 & 15,095 mars 1994 & 49426,595 & 0,693 & $-40,0$ & $+0,7$ & $+7,6$ & $+0,8$ \\
\hline 27 & 17,054 & 49428,554 & 0,955 & $+30,2$ & $-1,6$ & $-68,1$ & $+0,5$ \\
\hline 28 & 18,065 & 49429,565 & 0,090 & $+28,2$ & $+0,0$ & $-64,8$ & $-0,0$ \\
\hline 29 & 20,050 & 49431,550 & 0,355 & $-46,2$ & $-0,8$ & $+10,8$ & $-0,9$ \\
\hline 30 & 21,190 & 49432,690 & 0,508 & $-64,2$ & $-0,5$ & $+30,4$ & $-0,2$ \\
\hline 31 & 5,213 mars 1995 & 49781,713 & 0,152 & $+13,3$ & $+0,0$ & $-48,0$ & $+1,3$ \\
\hline 32 & 7,093 & 49783,593 & 0,403 & $-54,0$ & $+0,6$ & $+22,1$ & $+0,9$ \\
\hline 33 & 9,145 & 49785,645 & 0,677 & $-43,2$ & $+1,3$ & $+11,6$ & $+0,9$ \\
\hline 34 & 11,229 fev. 1996 & 50124,729 & 0,993 & $+36,0$ & $+0,5$ & $-73,0$ & $-0,6$ \\
\hline 35 & 12,227 & 50125,727 & 0,127 & $+20,2$ & $+0,2$ & $-56,2$ & $+0,1$ \\
\hline 36 & 14,185 & 50127,685 & 0,388 & $-51,7$ & $+0,4$ & $+18,4$ & $-0,2$ \\
\hline 37 & 18,090 avr. & 50191,590 & 0,929 & $+27,6$ & $+0,3$ & $-63,8$ & $+0,1$ \\
\hline 38 & 18,475 & 50192,475 & 0,047 & $+34,0$ & $-0,3$ & $-72,2$ & $-1,1$ \\
\hline 39 & 19,491 & 50193,491 & 0,183 & $+4,5$ & $+0,4$ & $-40,4$ & $-0,6$ \\
\hline 40 & 20,403 & 50194,403 & 0,305 & $-33,3$ & $-0,4$ & $-2,0$ & $-0,7$ \\
\hline 41 & 22,148 & 50195,648 & 0,471 & $-62,5$ & $-0,2$ & $+29,4$ & $+0,2$ \\
\hline 42 & 30,388 aout & 50326,388 & 0,943 & $+30,4$ & $+0,4$ & $-67,5$ & $+0,8$ \\
\hline 43 & 31,339 & 50327,339 & 0,070 & $+30,8$ & $-0,6$ & $-69,5$ & $-1,3$ \\
\hline 44 & 2,356 sep. & 50329,356 & 0,340 & $-41,2$ & $+0,8$ & $+9,2$ & $+1,1$ \\
\hline 45 & 28,207 nov. & 50415,707 & 0,880 & $+15,4$ & $-0,2$ & $-52,6$ & $-0,9$ \\
\hline 46 & 29,231 & 50416,731 & 0,017 & $+36,3$ & $+0,4$ & $-71,8$ & $+1,0$ \\
\hline 47 & 2,224 dec. & 50419,724 & 0,417 & $-57,1$ & $-0,4$ & & \\
\hline 48 & 29,152 jan. 1997 & 50477,652 & 0,159 & $+12,3$ & $+1,0$ & $-46,4$ & $+0,8$ \\
\hline
\end{tabular}

distance de 295 pc en très bon accord avec celle déduite de la parallaxe Hipparcos, $d=315(+92,-57)$ pc. Notons que dans notre étude nous avons négligé le rougissement interstellaire ; en effet, d'après les cartes de Lucke (1978), l'excès de couleur $E(B-V)$ ne devrait pas dépasser 0,04 mag.

\section{- Synchronisme rotation-révolution}

L'analyse du profil de la trace de corrélation permet d'accéder à la valeur de la projection de la vitesse équatoriale de chaque composante, $\nu \sin i$. Cette détermination, effectuée à l'observatoire de Genève, conduit ici à des valeurs très proches pour les deux composantes : $11,0 \mathrm{~km} / \mathrm{s}$ pour la primaire et $10,7 \mathrm{~km} / \mathrm{s}$ pour la secondaire, la précision sur ces quantités étant, pratiquement, de $1 \mathrm{~km} / \mathrm{s}$. Cette similitude, et la valeur courte de la période, permettent d'envisager le synchronisme entre rotation axiale et révolution orbitale au sein du système.

Dans cette hypothèse (et celle de coplanarité entre plans équatoriaux des étoiles et plan orbital), nous pouvons estimer le rayon des composantes par la relation : $R / R_{\odot}=0,01977 . P . V_{\mathrm{e}}$, où $V_{\mathrm{e}}$ est la vitesse équatoriale ; on obtient avec nos données $R \sim 2,85 \pm 0,25 R_{\odot}$ comme rayon moyen des composantes. Cette valeur étant 
compatible, tant avec celle trouvée plus haut qu'avec celles déduites directement de l'étude de systèmes similaires, mais présentant des éclipses (Kitamura \& Kondo 1978), nous pouvons donc retenir l'hypothèse du synchronisme.

\section{- Différence de magnitude et relation masse-luminosité}

Pour HD 125273, les éléments dont nous disposons : rapport de masses proche de l'unité et traces de corrélation de profils similaires, indiquent que l'étoile primaire et son compagnon sont de nature très voisine (vraisemblablement deux étoiles Am), ce qui nous permet de supposer $T_{2}=T_{1}$; la différence de magnitude des composantes peut alors être obtenue à partir des surfaces des traces de corrélation, $W_{1}$ et $W_{2}$, dont le rapport est proportionnel à celui des luminosités des deux étoiles (Lucke \& Mayor 1980). Comme les observations donnent $W_{1} / W_{2}=1,25$, on en déduit $\Delta m_{b}=\Delta m_{v}=0,24$ mag.

Dans une étoile binaire spectroscopique, il n'est en général pas possible de vérifier si les composantes, prises individuellement, satisfont ou non à la relation masseluminosité. Cependant, s'il s'agit d'une BS à deux spectres dont la différence de magnitude des composantes peut être estimée à partir des observations, on peut voir comment, globalement, le système se comporte vis-à-vis de cette relation. Pour effectuer ce test, nous avons utilisé la relation préconisée par Schmidt-Kaler (1982) pour les étoiles de la séquence principale $: \log M / M_{\odot}=0,46-0,10 M_{\mathrm{bol}}$, ce qui, dans le cas d'une binaire, s'écrit : $\log \left(M_{1} / M_{2}\right)=$ $0,10 \Delta M_{\mathrm{bol}}=0,25 \log \left(L_{1} / L_{2}\right)$. Cette relation, appliquée à HD 125273 , conduit à $L_{1} / L_{2}=1,17$ à partir du rapport de masses déduit des observations.

Le rapport des luminosités des composantes peut, d'autre part, être directement estimé à partir de la différence de magnitude $\Delta m_{v}$. En effet, compte tenu de l'hypothèse $T_{1}=T_{2}$, les corrections bolométriques sont les mêmes pour chaque composante du système et on a pratiquement : $\Delta m_{v}=\Delta M_{\mathrm{bol}}=2,5 \log \left(L_{1} / L_{2}\right)$, ce qui donne $L_{1} / L_{2}=1,25$. Cette dernière valeur étant proche, à moins de $10 \%$ près, de celle trouvée précédemment, nous en concluons que le système obéit à la relation masseluminosité, comme on pouvait s'y attendre vu le modèle proposé (système détaché, étoiles naines).

Remerciements. Nous tenons à remercier le referee, P. North, pour ses intéressantes suggestions et pour nous avoir fait bénéficier de ses connaissances dans l'utilisation et l'interprétation des indices de Strömgren. Nos remerciements aussi à M. Mayor pour l'attribution des missions à CORAVEL, ainsi qu'à S. Udry pour le traitement de nos observations à l'observatoire de Genève. Les recherches bibliographiques ont été effectuées à l'aide de la base de donnée SIMBAD du CDS de Strasbourg (France).

\section{Bibliographie}

Abt H.A., 1961, ApJS 6, 37

Abt H.A., 1965, ApJS 11, 429

Abt H.A., 1975, ApJ 195, 405

Abt H.A., 1984, ApJ 285, 247

Abt H.A., Bidelman W.P., 1969, ApJ 158, 1091

Abt H.A., Hudson K., 1971, ApJ 163, 333

Abt H.A., Snowden M.S., 1973, ApJS 25, 137

Abt H.A., Levy S.G., 1985, ApJS 59, 229

Baranne A., Mayor M., Poncet J.L., 1979, Vistas Astron. 23, 279

Batten A.H., Fletcher J.M., MacCarthy D.G., 1989, Publ. Dom. Astrophys. Obs. 17, 1

Bidelman, W.P., 1988, PASP 100, 1084

Conti P.S., Barker P.K., 1973, ApJ 186, 185

Crawford D.L., 1979, AJ 84, 1858

Dworetsky M.M., Moon T.T., 1986, MNRAS 220, 787

Ginestet N., Jaschek M., Carquillat J.M., Pédoussaut A., 1982, A\&A 107, 215

Hauck B., 1986, A\&AS 64, 21

Hauck B, 1992, Bull. Inform. CDS 40, 19

Kitamura M., Kondo M., 1978, Ap\&SS 56, 341

Lucke P.B., 1978, A\&A 64, 367

Lucke P.B., Mayor M., 1980, A\&A 92, 182

Michaud G., Tarasick D., Charland Y., Pelletier C., 1983, ApJ 269, 239

Moon T.T., Dworetsky M.M., 1985, MNRAS 217, 305

Nadal R., Ginestet N., Carquillat J.M., Pédoussaut A., 1979, A\&AS 35, 203

Narai K., 1971, PASJ 23, 529

North P., Jaschek C., Hauck B., Figueras F., Torra J., Künzli M., 1997, in: Procedings of the Hipparcos Venice ' 97 Symposium, ESA SP-402, p. 239

Olsen E.H., 1980, A\&AS 39, 205

Olsen E.H., 1983, A\&AS 54, 55

Schaller G., Schaerer D., Meynet G., Maeder A., 1992, A\&AS 96, 269

Schmidt-Kaler Th., 1982, in: Landolt-Börnstein, Zahlenwerte und Funktionen aus Naturwissenschaften und Technik, Gr.6, B.2, Springer-Verlag. Berlin, Heidelberg, New- York, p. 1 